AIAA-2004-1184

\title{
AN AEROACOUSTIC ANALYSIS OF WIND TURBINES*
}

\author{
Philip J. Morris, Lyle N. Long and Kenneth S. Brentner \\ Department of Aerospace Engineering \\ Penn State University, 233 Hammond Building \\ University Park, PA 16802

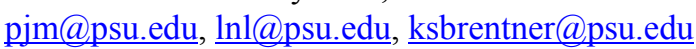

\begin{abstract}
This paper describes computational aeroacoustic methods that are being applied to predict the noise radiated by wind turbines. Since the wind turbine noise problem is very challenging, only some of the important noise sources and mechanisms are being considered. These are airfoil self-noise, the effects of blade rotation, and the propagation of sound over large distances. Two aspects of airfoil self-noise are being studied. The first is the relatively low frequency noise generated by deep stall and the second is trailing edge noise. The noise associated with blade rotation includes the effects of blade rotation on the blade aerodynamics, incoming gusts, incoming atmospheric turbulence and wind shear. The unsteady flow simulations are coupled to the radiated noise field with the permeable surface Ffowcs Williams - Hawkings formulation. For longrange noise propagation predictions, methods based on solutions of the linearized Euler equations or the Parabolic Equation approximation are discussed. Alternative methods for the implementation of boundary conditions for the propagation studies are also included.
\end{abstract}

\section{INTRODUCTION}

Wind turbines have aerodynamic and aeroacoustic behaviors with unique characteristics that make their prediction more challenging in many ways than already complicated aeroacoustic problems such as rotorcraft or propeller noise. In particular, the wind turbine blades can experience large changes in angle of attack associated with sudden large gusts, changes in wind direction, or interaction with the unsteady wake shed from the tower support on downwind, horizontal axis wind turbines. These blade/inflow/tower wake interactions can result in dynamic stall over portions of the rotating blade. These influences are described in reports based on the NREL "Unsteady Aerodynamics Experiments," e.g., see Robinson et al. [1].

\footnotetext{
*Copyright @ 2004 by Philip J. Morris, Lyle N. Long and Kenneth S. Brentner. Published by the American Institute of Aeronautics and Astronautics, Inc. and the American Society of Mechanical Engineers with permission.
}

The National Renewable Energy Laboratory (NREL) National Wind Technology Center (NWTC) has initiated a comprehensive, multi-year research program on the subject of wind turbine aeroacoustics. This involves experiments, modeling and simulation. One of the initiatives under this program is an attempt to incorporate new methods to analyze specific wind turbine noise sources. This includes the development of computational aeroacoustic (CAA) codes to analyze wind turbine blade noise sources and how they are impacted by key configuration variables. This paper describes the CAA techniques that have been developed by the authors in the past, including example applications, and how they will be applied to the study of wind turbine aeroacoustics.

It would be unrealistic to suggest that all aspects of the wind turbine noise problem could be simulated within the framework of a single aerodynamics/aeroacoustics code. The computational resources required to perform such a simulation will remain beyond the capabilities of available computers for many years. This can be easily demonstrated by consideration of the range of scales contained in complex wind turbine flows. These include the largest scales in vortex shedding from the support tower or in dynamic stall, to the finest scales of turbulence in the attached boundary layer. For example, at a Reynolds number of $1.1 \times 10^{7}$ the ratio of the smallest to the largest length scales is of order $2 \times 10^{5}$. Not only would such a simulation require an enormous number of grid points or cells, the execution time would require that the highest frequencies be resolved at the same time as sufficient time samples were being obtained to predict the lowest frequencies reliably. A more realistic and tractable approach is to separate the total problem into component problems and to apply the most efficient and appropriate numerical method to each sub-problem in turn. This is the approach described here. The particular noise issues to be examined involve the wind turbine airfoil self noise, the aerodynamics and aeroacoustics of rotating blades, and the propagation of sound, generated by the unsteady aerodynamics, through a varying atmosphere over an irregular terrain. Clearly, even these component aspects of the problem represent a significant computational challenge. The issues and numerical approaches being 
used are described for each of the aeroacoustic problems in the following sections.

\section{TECHNICAL APPROACH}

Each section of the technical approach provides a general description of the component noise problem and the methods being used for their simulation. This includes some examples of the use of these techniques by the authors in other aeroacoustic problems.

\section{Airfoil Self Noise}

Brooks et al. [2] categorized airfoil self noise into five source mechanisms:

- Turbulent boundary layer - trailing edge noise (TBL-TE)

- Laminar boundary layer - vortex shedding noise (LBL-VS)

- Boundary layer separation noise

- Large-scale separation (deep stall) noise

- Trailing edge bluntness - vortex shedding noise.

In addition, they considered noise generation associated with blade tip vortex formation. They also provided a semi-empirical prediction method based on available theory and experiment. Previous broadband noise prediction methods (see: Glegg et al. [3] and Grosveld [4]) have also used a semi-empirical approach. It should be recognized that these categories were developed for a non-rotating, symmetric airfoil (NACA 0012) in a two-dimensional flow. Clearly, the wind turbine noise problem is far more complex: but, these categories serve as a useful initial classification method. In this component of the technical approach the separation noise, including deep stall, and TBL-TE noise are considered.

A fundamental decision that must be made early in the project is whether the aerodynamic predictions should be made using an incompressible or compressible flow solver. For the relatively low Mach numbers of even the largest wind turbines the effect of compressibility on the unsteady flow is very small ${ }^{\ddagger}$. The inherent disadvantage of a compressible code is that the propagation speeds of the hydrodynamic and acoustic disturbances are widely separated. Thus the time step

* A $30 \mathrm{~m}$ diameter wind turbine rotating at $72 \mathrm{rpm}$ would have a tip velocity of $113 \mathrm{~m} / \mathrm{s}$ and a tip Mach number of approximately 0.33 . required to resolve the rapidly moving acoustic disturbances is much smaller than that required for the hydrodynamic fluctuations. This can result in inaccuracy or instability. This is not a problem for incompressible flow solvers. However, the incompressible solution contains no direct acoustic information, whereas the acoustics are an inherent part of the compressible solution. The disparity in propagation speeds can be overcome by preconditioning and dual time stepping (see: Merkle and Choi [5] and Buelow et al. [6]). If an incompressible solver is used to determine the surface pressures, it is possible to determine the acoustic radiation as long as a Green's function, which includes the effects of the surface geometry including the details of the airfoil trailing edge, is used. However, it is questionable whether any noise generated by aerodynamic noise sources away from the surface could be predicted accurately. Alternatively, a compressible solution would enable both the surface and volume sound sources to be included. Then, the far field could be obtained from the Ffowcs Williams-Hawkings (FW-H) extension [7] to the Lighthill acoustic analogy as described below. Based on the authors' experience in other aeroacoustic applications, the compressible flow solver approach is favored. This appears to be the appropriate choice for aerodynamic noise problems. However, this might not be the case for hydrodynamic noise problems where Mach numbers below as 0.01 are encountered.

It is important that the numerical aerodynamics approach be capable of predicting both attached and separated flows. Steady attached flows are predicted well by a Reynolds-averaged Navier-Stokes (RANS) solver with a turbulence model. However, even when the simulation is run in unsteady (ensemble-averaged) mode, such methods are found to be overly dissipative. Alternatively, separated flows are predicted quite well by Large Eddy Simulation (LES). A technique that incorporates the best aspects of both models is the Detached Eddy Simulation (DES). First developed for one-equation turbulence models (see Spalart et al. [8]), the technique can also be adapted to $k-\varepsilon$ and $k$ - $\omega$ models [see Strelets et al. [9]). The method depends on the choice of a turbulence length scale. In attached flow regions, the distance to the nearest wall determines the length scale. In separated flow, the length scale is set by the grid size. This provides an automatic transition between unsteady RANS and LES in separated flow. In the present research, a DES will be performed to predict the unsteady flow associated with deep stall. This provides the essential low frequency noise source information required to predict the acoustic radiation. 
Such deep stall flow separation noise is essentially low frequency. On the other hand, the noise generated by the interaction of the turbulent boundary layer and the airfoil trailing edge is of much higher frequency: being associated with the very small turbulence scales in the turbulent boundary layer. Though a DES is able to make predictions of separated flows at the largest scales, and their associated noise, the necessary grid resolution to describe the turbulent boundary layer perturbations is finer still. In the present research, a DES is used to provide a coarse simulation of the largescale unsteadiness of the flow for airfoils with both attached and mildly separated boundary layers. In turn, this provides an estimate of the long time-averaged flow properties.

The authors $[10,11]$ have performed a DES of both two- and three-dimensional cavity flows. The choice of a DES over a traditional unsteady RANS calculation using a $k-\varepsilon$ or $k-\omega$ model was made, since the traditional models had been found to be overly dissipative in the separated shear layer region. Since the development of the large-scale structures in the separated shear layer are an essential feature of the acoustic feedback process in a cavity flow, the traditional models typically give poor results. An interesting outcome of this study was the differences between the two- and three-dimensional simulations for shallow cavities: ones with a length to depth ratio $(L / D)$ greater than 4 . It was found that the two-dimensional simulations predicted the occurrence of a large, strong vortical structure that moved only slowly through the cavity. This has been referred to as a "wake-mode" [12]. However, no such behavior was observed when a three-dimensional simulation of the same $L / D$ cavity was performed. (The width to depth ratio was 1.) A comparison with experimental data [13] for the pressure coefficient on the floor of the cavity showed that the three-dimensional simulations gave good agreement, whereas the two-dimensional simulations gave very different predictions. This is shown in figure 1.

In order to simulate the finer scales in the boundary layer near the airfoil trailing edge the Non-Linear Disturbance Equations (NLDE) will be used. This technique, developed by the authors, (see Morris et al. [14]) is essentially a Large Eddy Simulation (LES) except that it solves for the perturbations about the assumed mean flow (provided in the present case by the DES). The advantage of this method over traditional LES for aeroacoustic applications is that it increases the accuracy of the solution in the acoustic field and also establishes a statistically stationary solution faster. This reduces the total computation time. The NLDE is used for the simulation of the flow in the trailing edge region

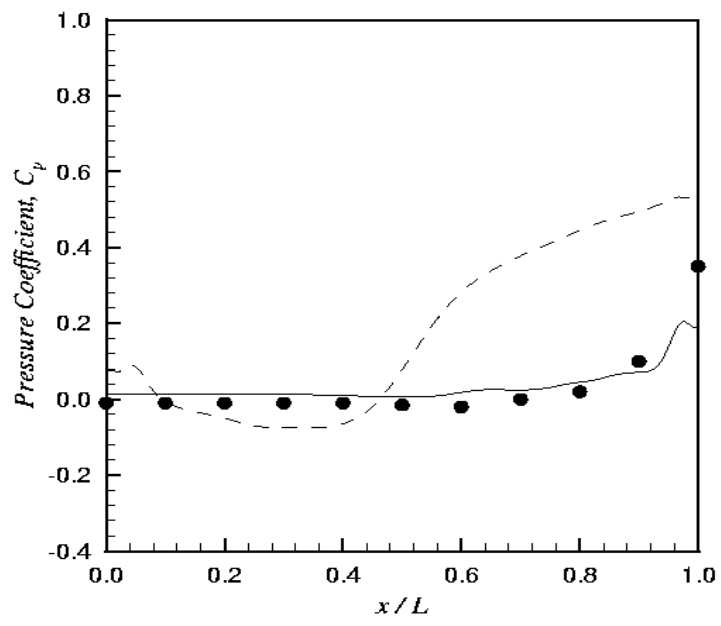

Figure 1. Comparison of the mean pressure coefficients on the cavity floor. $M=0.6$, $R e=200,000, L / D=4.4$. $\longrightarrow$, Three-dimensional cavity simulation $(W / D=1.0)$; $--_{-}-$: twodimensional cavity simulation; a : Experiment, Plentovich et al. [13].

of the airfoil. It predicts both fluctuations on the airfoil surface (surface pressure fluctuations) as well as the fine-scale turbulence in the vicinity of the blade trailing edge. In conjunction with the permeable surface FW-H equation method, these compressible flow simulations enable the associated far-field noise to be predicted. Sagaut et al [15] give an example of the use of the NLDE in a trailing edge flow problem.

Simulations based on the NLDE [14] have been performed by the authors in studies of the aerodynamics of bluff bodies [16] and jet aeroacoustics [17]. The focus of the jet noise studies was the noise of supersonic jets. This regime was chosen as it the largescale structures, which are the only ones that can be resolved at high Reynolds numbers, that provide the dominant noise contributions. The NLDE formulation provides good resolution in the radiated noise field, where the density and pressure perturbations are very small in comparison to the corresponding mean properties. Figure 2 shows the predicted contours of equal sound pressure level for an unheated supersonic jet with exit Mach number, $M_{j}=2.1$. The strong directional radiation to approximately $45^{\circ}$ to the jet downstream axis is characteristic of supersonic jet noise and is associated with the supersonic convection of large-scale structures in the jet shear layer. 


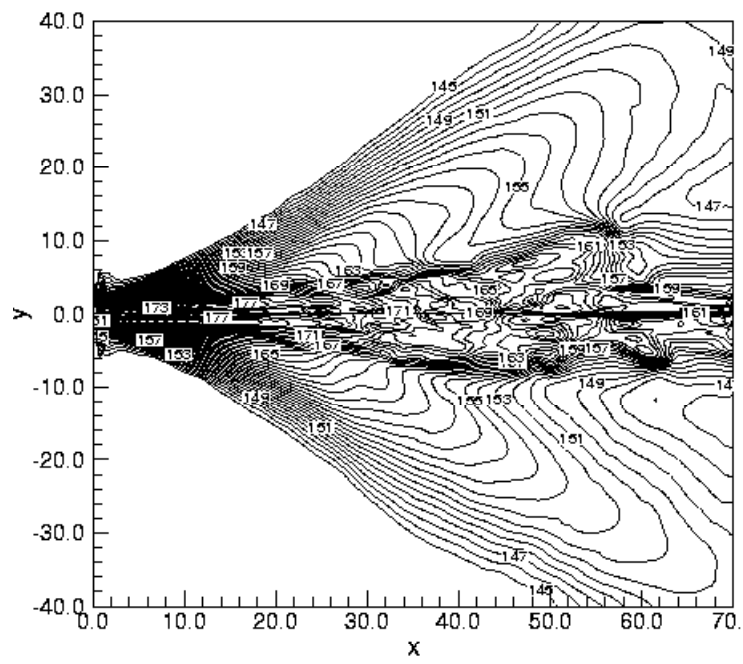

Figure 2. Contours of equal sound pressure level for an unheated axisymmetric $M_{j}=2.1$ jet.

It is important to note that, at high Reynolds numbers, the highest frequencies expected in broadband airfoil noise would exceed those resolved by even the NLDE or LES. They fall into the range of scales modeled by a sub-grid scale turbulence model. This is an inherent problem with LES for aeroacoustics (unless the Reynolds number is so low that the LES is really a Direct Numerical Simulation). However, aeroacoustic models based on solutions to the averaged equations have been used for noise predictions in other branches of aeroacoustics. Thus, predictions of the sub-grid scale noise would have to be made based on a statistical model similar to those developed by Tam and Auriault [18] and Morris and Farassat [19]. The authors are involved in the further development of such models for jet noise applications and these developments will be transitioned to the broadband wind turbine noise problem as needed.

All of the codes being developed for the wind turbine airfoil self-noise predictions described in this section are based on an existing code developed by the authors. Called the Compressible, High-Order, Parallel, Acoustics (CHOPA) code, it is written in Fortran 90 with MPI for use on distributed memory computers. It is based on an explicit 4th-order Runge-Kutta timemarching scheme with 4th-order Dispersion-RelationPreserving spatial discretization. The code has been used in previous and on-going studies of gust-airfoil interactions, cavity noise, nonlinear acoustics, and jet aeroacoustics.

\section{$\underline{\text { Rotating Blades }}$}

We have been investigating the aerodynamics and aeroacoustics of rotating blades. While important information can be learned from two-dimensional and non-rotating simulations, other aspects of the physics of sound from wind turbines must be obtained from rotating blade simulations. As shown by Corfeld et al. [20] the three-dimensional flow over rotating blades can be significantly different than the flow over a wing, and there can also be dramatic differences between 2-D and 3-D simulations. Rotating blades can have significant spanwise (or radial) flow. Also of course, the blade speed varies linearly from root to tip. In addition, the three-dimensional wake of a rotating blade often remains in close proximity to the blade for a long period of time (compared to the wake of a wing). For these reasons we are performing compressible threedimensional rotating blade simulations. On the basis of our results from previous experiences, a compressible flow solver is being used.

We are using a range of methods from inviscid to largeeddy simulation (LES). Using the time-dependent, compressible, governing equations will allow us to simulate a number of important phenomena:

- Incoming gusts

- Incoming atmospheric turbulence

- Wind shear

These are important for leading edge noise and tip noise. By incorporating time dependent boundary conditions, we can introduce either a gust or turbulent incoming flow. We have done this in the past (Chyczewski et al. [21]) with jet noise predictions, to simulate turbulence levels inside the nozzle and their effect on the jet shear layers and noise. This method can be used to study leading edge noise due to gusts and turbulence inflow. We can also put in larger effects such as the simulation of the effect of having a wind turbine in an atmospheric boundary layer. The code we use has been under development at Penn State for more than six years. It is an unstructured-grid finite-volume solver called PUMA2, and it has the capability to simulate rotating blades through the use of additional metric terms in the equation that incorporate grid velocities into the flux terms [22]. Figure 3 illustrates some preliminary results using an unstructured rotating grid CFD code for a two-bladed rotor with an NACA 0012 airfoil. Isosurfaces of vorticity are shown. The Mach number on the surface is shown in Figure 4. 


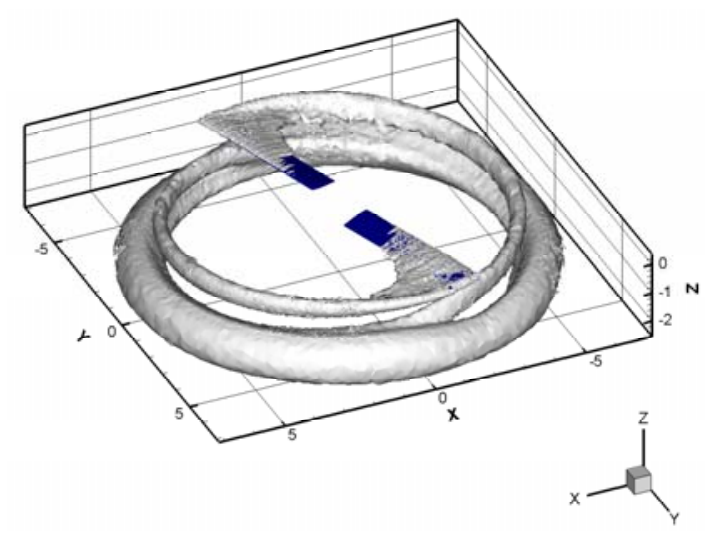

Figure 3 Iso-surfaces of vorticity on rotating blade.

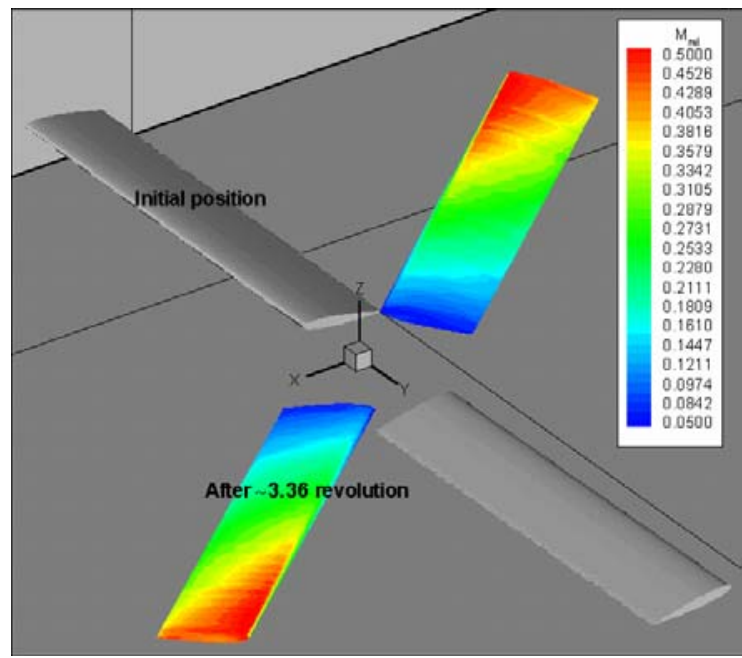

Figure 4. Surface Mach numbers on rotating blade.

We have also begun simulating dynamic stall phenomena [22]. Figures 5 and 6 show pressure contours and streamlines around a finite wing with an NACA 0015 airfoil oscillating in pitch. The Mach number of this flow is 0.29 , and we will be comparing to experimental data soon. These are also 3-D simulations.

Some unstructured solvers can be quite dissipative due to the use of upwind Riemann solvers, however we have the capability to use a central difference scheme for the flux calculations, which minimizes the dissipation. In addition, through the use of unstructured grids, we are able to heavily cluster points in regions of interest. We have also incorporated a sub-grid scale

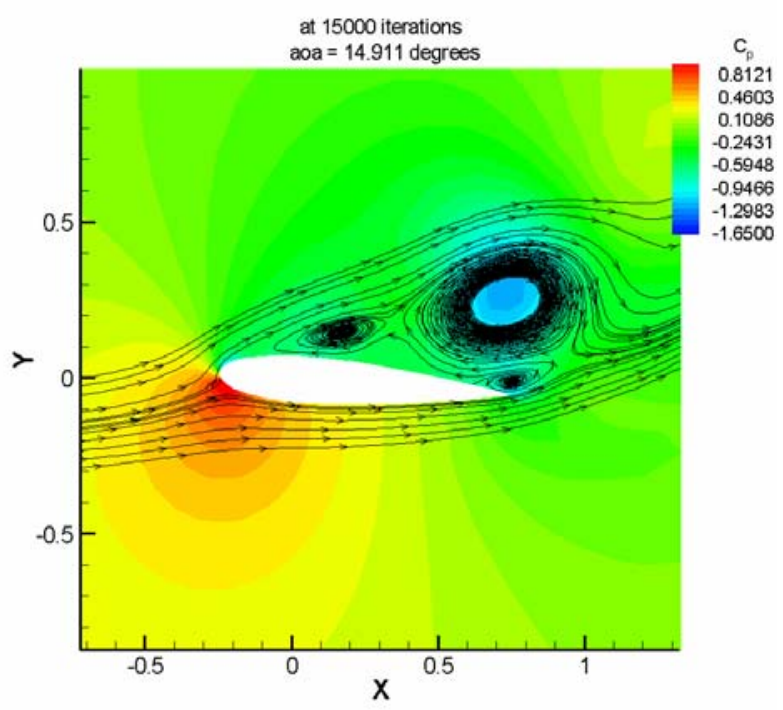

Figure 5. NACA airfoil pitching up at 14.9 degrees.

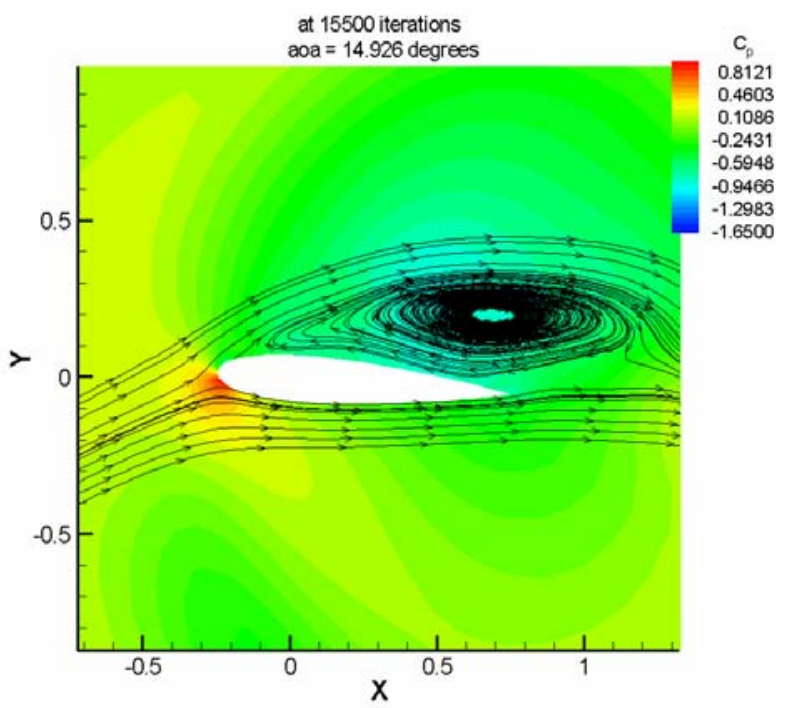

Figure 6. NACA airfoil pitching up at 14.9 degrees.

LES turbulence model, and have performed timeaccurate simulations of complete helicopter fuselage flow fields (Souliez and Long [23]), as well as a complete time-accurate simulation of turbulent flow over a landing gear and its radiated noise (Souliez et al. [24]). The time step sizes need to be small, but this is a very fast code written in C/MPI, which runs on Beowulf clusters and massively parallel machines (e.g. IBM SP and SGI). We can easily run hundreds of 
thousands of time steps in a few days on a small 16processor Beowulf machine. It is essential to perform some simulations that incorporate all of the physics of the unsteady problem. This code can quite easily simulate incoming unsteady flow fields such as wakes, atmospheric turbulence, large gusts, or atmospheric shear layers; and the radiated noise due to those effects. In this code we use efficient Gauss-Seidel or SOR methods to compute a steady-state field, and then turn on time-accurate Runge-Kutta time marching.

Since these will be compressible time-accurate simulations, they can be coupled directly to a FW-H code to predict radiated far-field noise, as described in the next section. As the simulation runs, we occasionally store data on a permeable surface in the flow field. This data can then be post-processed to provide the far-field noise. We have found that the FW-H approach has a wider range of applicability and requires very little additional work compared to a Kirchhoff method.

\section{$\underline{\text { Radiation and Propagation }}$}

The radiation and propagation of noise from the source region to a distant observer is the unifying component in the wind turbine noise prediction system. The FW$\mathrm{H}$ equation will be the primary tool for noise prediction in the intermediate- and far-fields. The FW-H equation is the most general form of the Lighthill acoustic analogy and is appropriate for the prediction of rotating blade noise both in either the time or frequency domain. The FW-H equation is an exact rearrangement of the Navier-Stokes equations into the form of an inhomogeneous wave equation with surface (monopole and dipole) and volume (quadrupole) source terms. The FW-H equation can be written as:

$$
\begin{aligned}
\square^{2} p^{\prime}(\mathbf{x}, t)= & \frac{\partial}{\partial t}\left\{\left[\rho_{o} v_{n}+\rho\left(u_{n}-v_{n}\right)\right] \delta(f)\right\} \\
& -\frac{\partial}{\partial x_{i}}\left\{\left[\Delta P_{i j} \hat{n}_{j}+\rho u_{i}\left(u_{n}-v_{n}\right)\right] \delta(f)\right\} \\
& +\frac{\bar{\partial}^{2}}{\partial x_{i} \partial x_{j}}\left[T_{i j} H(f)\right]
\end{aligned}
$$

where $\rho_{o}$ is the density of the undisturbed fluid, $u_{n}$ is the fluid velocity in the direction normal to the integration surface (defined by $f=0$ ), $v_{n}$ is the normal velocity of the integration surface, $\Delta P_{i j}$ is the jump in the compressive stress tensor across the integration surface, and $T_{i j}$ is the Lighthill stress tensor. The surface sources (identified by the presence of the Dirac

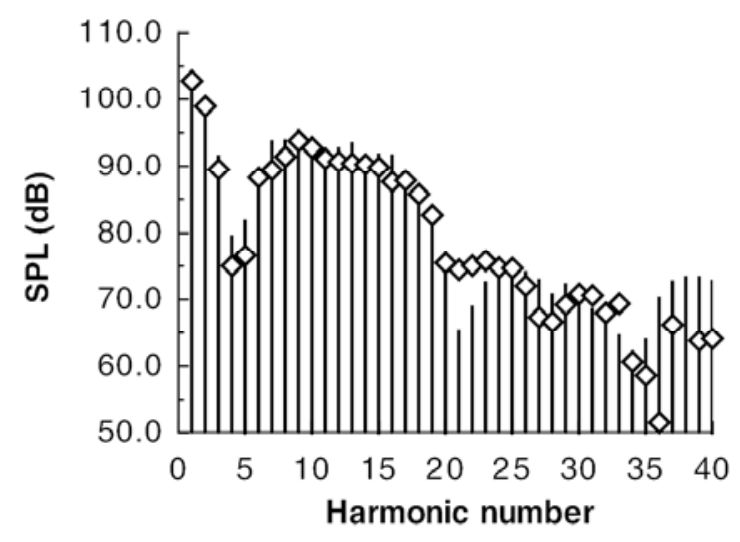

Figure 3. Comparison of measured $\diamond$ and predicted | sound pressure level (SPL) for a model rotor operating in a BVI noise condition.

delta function, $\delta(f))$ can be specified either on a surface corresponding to the actual blade surface or a fictitious data surface that contains all of the desired physical noise sources (Brentner and Farassat [25]). Because the fictitious surface is located in the flow field, this form of the FW-H equation is usually called a porous or permeable surface $\mathrm{FW}-\mathrm{H}$ formulation. In either permeable or actual blade surface application, the source strength is determined a priori through the computational methods described in the previous sections.

The FW-H equation has been used extensively to predict rotorcraft (e.g., Brentner [26]; Brentner and Farassat [27]; Farassat and Brentner, [25]; Brentner, [28]). In particular, Brentner [26] has developed a series of rotor noise prediction codes, known as WOPWOP, to predict the noise from helicopter rotors and tiltrotors. WOPWOP noise predictions have been validated for thickness, loading, high-speed impulsive, and blade-vortex-interaction (BVI) noise predictions both with measured and computed source strength data. Geometrical details, such as airfoil shape, blade geometry can be fully represented in the numerical implementation and the sensitivity of the acoustic predictions to modeling parameters has been documented by Brentner et al. [29]. Figure 3 (from Ref. [30]) shows the excellent agreement that is possible for the difficult BVI noise condition when measured blade pressure data are used as input to the noise prediction. The discrete frequency noise prediction agrees very well with the measured data up to the $35^{\text {th }}$ harmonic. This case demonstrates that if blade loading is known accurately, then the $\mathrm{FW}-\mathrm{H}$ 
equation will predict the noise accurately as well. A primary challenge for wind turbine noise prediction is the accurate prediction of the unsteady blade loading.

Recently a new rotor noise prediction code, known as PSU-WOPWOP (see Brentner et al., [31], [32]), has been developed to compute the noise of rotorcraft in maneuvering flight. This code can predict the noise of rotor blades in arbitrary motion with unsteady, aperiodic loading — both with solid or permeable data surfaces. This combination of features (especially aperiodic loading and permeable surface formulation) make PSU-WOPWOP well suited as the platform for wind turbine noise prediction (including the effects of gusts and time-dependent wind unsteadiness). An enhancement of PSU-WOPWOP will support both the airfoil self noise and rotating blade source noise developments of the previous sections. Furthermore, two-dimensional computations can readily be put into a rotating frame by assuming the properties applicable to a strip along the rotating blade radius.

Although much work has been done to develop and validate the $\mathrm{FW}-\mathrm{H}$ method for rotors, the $\mathrm{FW}-\mathrm{H}$ approach has also been applied successfully to both two-dimensional and three-dimensional model problems with significant flow field turbulence, including vortex shedding from a circular cylinder (Cox et al. [33]), trailing edge noise scattering (Singer et al. [34]) and the analysis of slat trailing edge noise (Singer et al. 35). In these studies, it was demonstrated that the FW-H equation predicts the correct directivity and velocity scaling for the trailing edge scattering problem, provided that compressible flow field data is used on the data surface.

Until recently, the $\mathrm{FW}-\mathrm{H}$ approach has been used primarily to predict discrete frequency noise, such as that from rotating blades. While discrete frequency noise is certainly an important component of wind turbine noise (especially at low frequencies), broadband noise sources are also very important (especially at the higher frequencies). In particular, Singer et al. [34] predicted the noise from a simulated turbulent flow past the trailing edge of an airfoil. A high resolution, unsteady inviscid flow calculation was performed with a small flat plate inserted above the upper surface of the airfoil at the $98 \%$ chord location. The airfoil was a $2.6 \%$ NACA 00 series airfoil and the flat plate was perpendicular to the flow. Numerical viscosity in the computation resulted in the shedding of vortices from the plate, which then convected past the trailing edge of the airfoil. Figure 7 shows instantaneous vorticity

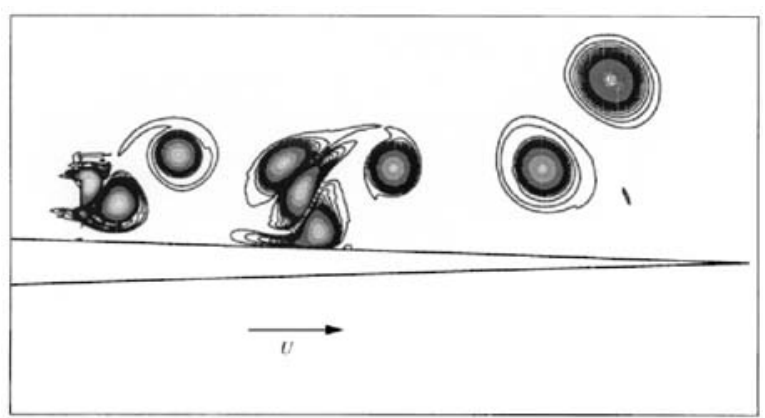

Figure 7. Instantaneous vorticity magnitude contours in vicinity of trailing edge for $M=0.2$ case. Approximately $2 \%$ of the aft portion of airfoil is shown.

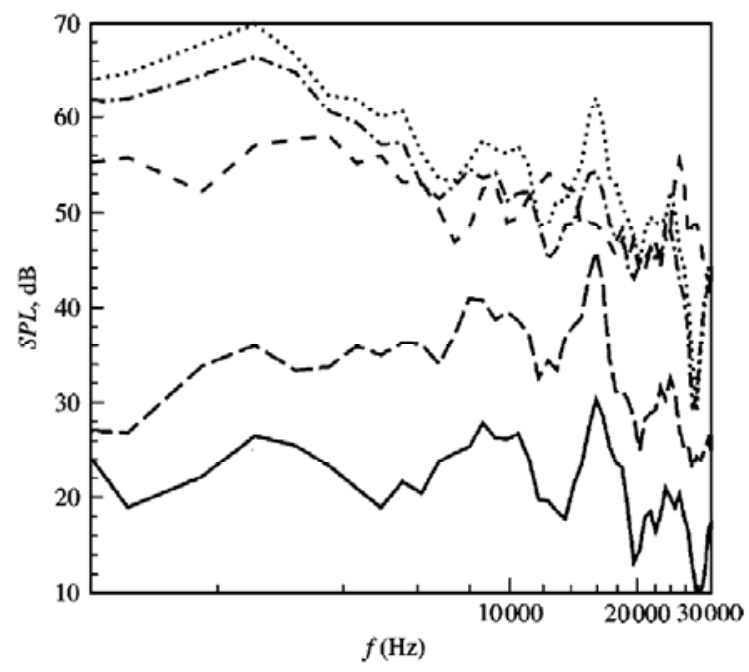

Figure 8. Spectra of acoustic signals (referenced to $20 \mu \mathrm{Pa}$ ) for observers located $10 \mathrm{C}$ from trailing edge of airfoil; on -airfoil-body integration surface used, $M=0.2$. Observers located at: $\longrightarrow, 0^{\circ} ;----, 45^{\circ} ;-\cdot-\cdot-, 90^{\circ} ; \cdots \cdots, 135^{\circ}$; $-\longrightarrow, 180^{\circ}$.

magnitude contours for the case when the freestream flow was $M=0.2$.

The unsteady pressure field on both the airfoil surface and several off-body $\mathrm{FW}-\mathrm{H}$ surfaces were used to predict the sound from the trailing edge scattering noise (shown in figure 8). Singer et al. [34] demonstrated for this example problem that the directivity of the noise was similar to that of a noise source traveling past a semi-infinite plate and that the velocity scaling agreed with that expected for the trailing edge noise scattering problem. It is also apparent that the noise predicted in 
this computation is largely broadband in nature. Hence, the FW-H approach is able to predict both the discrete frequency noise and broadband noise components of wind turbines - once the unsteady loading is computed successfully.

It has also been shown that the permeable surface FW$\mathrm{H}$ formulation can be used in a diagnostic manner by judicial placement of the data surface around a limited portion of the source region to determine the noise contributed by that limited region (Singer et al. [34], [35]). This capability has the potential to enable blade designers to examine noise generated by small regions of the flow, but a deeper understanding of how much cancellation of noise occurs between neighboring source regions is needed to fully utilize this capability. Finally, Casper and Farassat [36], have proposed a new formulation of the $\mathrm{FW}-\mathrm{H}$ equation, which they call formulation $1 \mathrm{~B}$, for the prediction of broadband noise sources. They have verified that this formulation predicts the correct velocity scaling for both compact and noncompact sources. In particular, the predicted noise due to turbulence interacting with a NACA 0012 gives good agreement with the experiment of Patterson and Amiet [37] over a range of forward speeds.

The typical application of the FW-H equation is based on the assumption that the sound radiates into an unbounded domain. However, the sound generated by wind turbines, particularly the low frequency components, may propagate large distances through an unsteady, nonuniform atmosphere over an irregular terrain. Atmospheric absorption can also be significant for the high frequency noise components. Thus, for wind turbine applications, sound propagation is an important component of the complete aeroacoustic problem. The effect of acoustic propagation will be predicted by a numerical solution of the linearized Euler equations (LEE) or methods adapted from underwater acoustics and electromagnetics.

The use of the LEE enables any effects due to mean velocity and temperature gradients to be included. The sound sources can be specified on a data surface surrounding the wind turbine. Then there are several ways in which the sound propagation problem could be solved. The first would involve the numerical solution of the LEE in the time domain with the pressures and velocities specified on the data surface. However, a new solution would be required for every new source specification. Alternatively, the Green's function for the particular geometry, wind and temperature conditions could be determined numerically. However, this would require a different Green's function for every point on the data surface. This approach would be very inefficient. This inefficiency can be overcome by posing the adjoint LEE problem. In this case, a sound source is placed at selected observer locations and sound is propagated back towards the data surface. This adjoint solution is identical to the Green's function for any surface point that radiates to the selected observer location. Thus, the Green's function for every surface point is obtained in a single calculation. Tam and Auriault [38] introduced this technique for the jet noise radiation problem. This formulation is not without problems as hydrodynamic instabilities, which would be limited physically by nonlinear effects, can be triggered and can overwhelm the acoustic solution. Agarwal et al. [39] have developed a frequency domain solution method that overcomes this problem.

Since the long-range acoustic propagation problem is concerned primarily with low frequencies and long wavelengths, wave propagation techniques based on the Parabolic Equation (PE) approximation are attractive. Originally introduced for electromagnetic problems by Lentovich and Fock [40], it was introduced to the ocean acoustics community by Tappert[41]. Lee et al. [42] give an excellent review of the PE development in the twentieth century. The method is based on the Helmholtz equation that is approximated in the far field by a one-way propagation equation of parabolic form. This means that the solution can be marched in the range direction. The method is very efficient for longrange, low frequency, wave propagation with negligible backscatter. As such, it appears to be well suited to the wind turbine noise propagation problem. The authors are exploring a novel implementation of the PE method in adjoint form.

An additional difficulty that arises is the specification of the boundary condition that describes the ground surface. This could be a reflecting or partially absorbing surface. In addition, the surface is likely to be very irregular. The authors have developed a technique, the Impedance Mismatch Method (IMM) [43], which enables an arbitrary geometry surface to be specified while retaining a simple Cartesian grid geometry. The authors have also used the Brinkman penalization method [44] for acoustic scattering and blast loading simulations. This formulation is similar in nature to the IMM though it appears to be more stable for higher amplitude waves and is computationally slightly less expensive. Finally, it is encouraging to note that the use of a frequency domain solution for the propagation problem, as indicated above, would be very compatible with the specification of different complex impedance surface boundary conditions. 


\section{CLOSING REMARKS}

This paper has described several CAA techniques that offer considerable promise for the prediction of noise from wind turbines. All the techniques focus on the prediction of the unsteady aerodynamics of wind turbines. Several CAA codes with different formulations are being used. They include structured and unstructured grid methods and time and frequency domain formulations. The choice of methodology is

\section{REFERENCES}

1 Robinson, M. C., Hand, M. M., Simms, D. A. and Schreck, S. J. 1999, "Horizontal Axis Wind Turbine Aerodynamics: Three-Dimensional, Unsteady, and Separated Flow Influences," NREL/CP-500-26337. Presented at the $3^{\text {rd }}$ ASME/JSME Joint Fluids Energy Conference, San Francisco, CA.

2 Brooks, T. F., Pope, D. S. and Marcolini, M. A., 1989, "Airfoil Self-Noise and Prediction," NASA Reference Publication 1218.

3 Glegg, S. A. L., Baxter, S. M. and Glendenning, A. G. 1987, "The Prediction of Broadband Noise From Wind Turbines," Journal of Sound and Vibration, 118(2), pp. 217-239.

4 Grosveld, F. W. 1985, "Prediction of Noise From Horizontal Axis Wind Turbines," AIAA Journal of Propulsion, 1(4), pp. 292-299.

5 Merkle, C. L. and Choi, Y.-H. 1988, "Computation of Low-Speed Compressible Flows With Time-Marching Procedures," Intl. Journal for Numerical methods in Engineering, 25, pp. 293-311.

6 Buelow, P. E. O., Schwer, D. A., Feng, J., Merkle, C. L. and Choi, Y.-H. 1997, "A Preconditioned DualTime, Diagonalized ADI Scheme For Unsteady Computations," AIAA Paper No. 97-2101.

7 Ffowcs Williams, J. E. and Hawkings, D. L. 1969, "Sound Generation by Turbulence and Surfaces in Arbitrary Motion," Phil. Trans. of the Royal Society of London, Series A, 264(1151), pp. 321-342.

8 Spalart, P. R., Jou, W.-H., Strelets, M. and Allmaras, S. R. 1997, "Comments on the Feasibility of LES for Wings and on a Hybrid RANS/LES Approach," In Proceedings of the First AFOSR International dictated by the component problem under investigation. The unsteady flow information is coupled to the radiated noise using the permeable surface FW-H formulation. This is the unifying concept of the study. Each of the component aeroacoustic problems under investigation is very challenging. However, it is anticipated that the suite of techniques and codes under development will provide a very powerful tool for the prediction of wind turbine noise.

Conference on DNS/LES, Ruston, LA. Greyden Press, Columbus, $\mathrm{OH}$.

9 Strelets, M. 2001, "Detached Eddy Simulation of Massively Separated Flows," AIAA Paper No. 20010879 .

10 Shieh, C. M. and Morris, P. J. 2000, "Parallel Computational Aeroacoustic Simulation of Turbulent Subsonic Cavity Flow," AIAA Paper No. 2000-1914.

11 Shieh, C. M. and Morris, P. J. 2001, "Comparison of Two- and Three-Dimensional Turbulent Cavity Flows," AIAA Paper No. 2001-0511.

12 Rowley C. W., Colonius, T. and Basu, A. J. 2002, "On Self-Sustained Oscillations in Two-Dimensional Compressible Flow Over Rectangular Cavities," Journal of Fluid Mechanics, 455, pp. 315-346.

13 Plentovich, E. B., Stallings Jr., R. L. and Tracey, M. B. 1993, "Experimental Cavity Pressure Measurements at Subsonic and Transonic Speeds," NASA Technical Memorandum 4363.

14 Morris, P. J., Long, L. N., Bangalore, A. and Wang, Q. 1997, "A Parallel Three-Dimensional Computational Aeroacoustics Method Using Nonlinear Disturbance Equations," J. of Computational Physics, 133, pp. 5774.

15 Sagaut, P., Garnier, E., Tromeur, E., Larchevêque, L. and Labourasse, E. 2003, "Turbulent Inflow Conditions for LES of Supersonic and Subsonic Wall Bounded Flows," AIAA Paper No. 2003-0068.

16 Hansen, R. P., Long, L. N. and Morris, P. J. 2000, "Unsteady, Laminar Flow Simulations Using the Nonlinear Disturbance Equations," AIAA Paper No. 2000-1981. 
17 Morris, P. J., Long, L. N., Scheidegger T. E. and Boluriaan, S. 2002, "Simulations of Supersonic Jet Noise,' International Journal of Aeroacoustics, 1(1), pp. 17-42.

18 Tam, C. K. W. and Auriault, L. 2000, "Jet Mixing Noise From Fine-Scale Turbulence," AIAA Journal, 37(2), pp. 145-153.

19 Morris, P. J. and Farassat, F. 2002, "Acoustic Analogy and Alternative Theories for Jet Noise Prediction," AIAA Journal, 40(4), pp. 671-680.

20 Corfeld, K., Strawn, R., and Long, L.N. 2002, “A Computational Aerodynamics Simulation of a Martian Rotorcraft," submitted to Journal of the American Helicopter Society.

21 Chyczewski, T., Long, L. N., and Morris, P. 1998, "Numerical Study of Nozzle Exit Condition Effects on Jet Development," AIAA Journal, 36(6), pp. 986-993.

22 Sezer-Uzol, N., "Simulations of Unsteady Separated Flows," Ph.D. Thesis, Aerospace Engineering, Penn State Univ., expected Aug., 2004.

23 Souliez, F. and Long, L. N. 2002, “Computational Simulations of Rotorcraft Fuselage Drag," submitted to the Journal of the American Helicopter Society.

24 Souliez, F., Long, L. N., Morris, P.J., and Sharma, A. 2002, "Landing Gear Aerodynamic Noise Prediction Using Unstructured Grids," International Journal of Aeroacoustics, 1(2), pp. 115-136.

25 Brentner, K. S. and Farassat, F. 1998, “Analytical Comparison of The Acoustic Analogy and Kirchhoff Formulation for Moving Surfaces," AIAA Journal, 36(8), pp. 1379-1386.

26 Brentner, K. S. 1986, "Prediction of Helicopter Discrete Frequency Noise - a Computer Program Incorporating Realistic Blade Motions and Advanced Acoustic Formulation," NASA TM-87721.

27 Brentner, K. S. and Farassat, F. 1993, "Helicopter Noise Prediction: the Current Status and Future Direction," Journal of Sound and Vibration, 170(5), pp. 79-96.

28 Brentner, K. S. 2000, "Modeling Aerodynamically Generated Sound: Recent Advances in Rotor Noise Prediction," AIAA Paper No. 2000-0345.
29 Brentner, K. S., Marcolini, M. A., and Burley, C. L. 1994, "Sensitivity of Acoustic Prediction to Variation of Input Parameters," Journal of the American Helicopter Society, 39(3), pp. 43-52.

30 Brentner, K. S., and Farassat, F. 1994, "Helicopter Noise Prediction: the Current Status and Future Direction," Journal of Sound and Vibration, 170(5), pp. 79-96.

31 Brentner, K. S., Brès, G. A., Perez, G., and Jones, H. E. 2002, "Maneuvering Rotorcraft Noise Prediction: a New Code for a New Problem," presented at the AHS Aerodynamics, Acoustics, and Test Evaluation Technical Specialists Meeting, San Francisco, CA, January 23-25.

32 Brentner, K. S., Perez, G., Brès, G. A., and Jones, H. E. 2002, "Toward a Better Understanding of Maneuvering Rotorcraft Noise," American Helicopter Society 58th Annual Forum Proceedings, Montreal, Canada, June 11-13.

33 Cox, J. S., Brentner, K. S., and Rumsey, C. L. 1998, "Computation of Vortex Shedding and Radiated Sound for a Circular Cylinder: Subcritical to Transcritical Reynolds Numbers," Theoretical and Computational Fluid Dynamics, 12(3), pp. 233-253.

34 Singer, B. A., Brentner, K. S., Lockard, D. P. and Lilley, G. M. 2000, "Simulation of Acoustic Scattering From a Trailing Edge," Journal of Sound and Vibration, 230(3), pp. 541-560.

35 Singer, B.A., Lockard, D. P., and Brentner, K. S. 2000, "Computational Aeroacoustics Analysis of Slat Trailing-Edge Flow,” AIAA Journal, 38(9), pp. 15581564.

36 Casper, J. and Farassat, F. 2002, "A New Time Domain Formulation for Broadband Noise Prediction," International Journal of Aeroacoustics, 1(3), pp. 207240 .

37 Patterson, R. W. and Amiet, R. K. 1977, "Noise and Surface Pressure Response of an Airfoil to Incident Turbulence," Journal of Aircraft, 14(8), pp. 729-736.

38 Tam, C. K. W. and Auriault, L. 1998, "Mean Flow Refraction Effects on Sound Radiated From Localized Sources in a Jet," Journal of Fluid Mechanics, 370, pp. 149-174. 
39 Agarwal, A., Morris, P. J. and Mani, R. 2003, "The Calculation of Sound Propagation in Nonuniform Flows: Suppression of Instability Waves," AIAA Paper No. 2003-0878.

40 Lentovich, M. A. and Fock, V. A. 1946,"Solution of Propagation of Electromagnetic Waves Along the Earth's Surface by the Method of Parabolic Equations," Journal Physics USSR, 10, pp. 13-23.

41 Tappert, F. D. 1977, "The Parabolic Equation Approximation Method," in Wave Propagation and Underwater Acoustics, eds. J. B. Keller and J. S. Papadakis, Lecture Notes in Physics, 70, SpringerVerlag, Heidelberg.

42 Lee, D., Pierce, A. D. and Shang, E.-C. 2000, "Parabolic Equation Development in the Twentieth Century," Journal of Computational Acoustics, 8(4), pp. 527-637.

43 Chung, C. and Morris, P. J. 1998, "Acoustic Scattering From Two- and Three-Dimensional Bodies," J. Computational Acoustics, 6(3), pp. 357-375.

44 Brinkman, H. C. 1947, "A Calculation of the Viscous Force Exerted by a Flowing Fluid on a Dense Swarm of Particles," Applied Scientific Research, A1, pp. 354-366. 